American Journal of Infectious Diseases 4 (3): 200-203, 2008

ISSN 1553-6203

(C) 2008 Science Publications

\title{
Epstein-Barr Virus Infection as A Trigger of Autoimmune Hepatitis: Case Report
}

\author{
${ }^{1}$ D. Cabibi, ${ }^{2}$ S. Scaduti, ${ }^{1}$ M. Cacciatore and ${ }^{1}$ F. Di Gaudio \\ ${ }^{1}$ Department of Human Pathology, University of Palermo, Italy \\ ${ }^{2}$ Service of Hepatology, Hospital R. Guzzardi Vittoria (Ragusa), Italy
}

\begin{abstract}
The study underlines the importance of the differential diagnosis between primary EpsteinBarr virus EBV associated hepatitis with features of autoimmunity, in which there is a direct pathogenetic role of the virus, and EBV related autoimmune hepatitis, in which EBV could act as the trigger of the immune-mediated damage with probable differences between the two conditions with regard to the prognosis and the responsiveness to immunosuppressive treatment. Moreover we hypothesise that the favourable outcome in our patient, better than the most of autoimmune hepatitis cases, may be related either to the moderate necroinflammatory activity and to the low level of fibrosis at the beginning of the disease or to the role of EBV as a trigger of autoimmune hepatitis. The hypothesis that EBV-related autoimmune hepatitis could have a more favourable prognosis than the most of autoimmune hepatitis cases in general need to be confirmed on a larger series of studies.
\end{abstract}

Key words: AIH, EBV, HEV, outcome

\section{INTRODUCTION}

Several hepatotropic viruses have been postulated as inducers of autoimmune hepatitis (AIH) in susceptible subjects ${ }^{[1-5]}$. The Epstein-Barr virus (EBV) is among these agents ${ }^{[6-9]}$ but the number of patients reported is small and, to our knowledge, there is little information on the clinical evolution of the disease in such patients. The present report describes a young man that, 16 months after the recovery of acute hepatitis E, was infected with EBV and 4 months after developed autoimmune hepatitis.

\section{CASE REPORT}

A 31 years old male was admitted to hospital in May 2000 with jaundice and malaise. Since about one week, it was noticed dyspeptic illness (gastric pyrosis, sensation of abdominel tension) and successively issue of hypochromic faeces and hyperchromic urines. Aminotransferases were markedly increased (AST/ALT = 935/1400 UI/l, normal range 10-45 UI/l for AST and 10-43 UI/l for ALT) Serum bilirubin was $5 \mathrm{mg} / \mathrm{dl}$. Ceruloplasmin and alpha-1-antitrypsin were within normal limits. There was no history of hepatotoxic drugs. Anti-HAV, anti-HBV, anti-HCV and autoantibodies (ANA, SMA, AMA) were negative. Anti-HEV was positive, and a diagnosis of acute hepatitis type $\mathrm{E}$ was made. A liver biopsy showed acute hepatitis. Parenchymal damage was most severe in perivenular areas. Features included spotty necrosis, livercell drop-out and infiltration by lymphocytes and macrophages. Macrophages contained ceroid pigment, staining with PAS after diastase digestion (Fig. 1A).

Reticulin framework was condensed near terminal venules, but not near portal tracts indicating perivenular collapse of the parenchima, like an acute hepatitis. Shikata orcein staining, that is useful to distinguish between recent collapse of acute hepatitis, lacking of detectable elastic fibres, and old fibrosis that usually stains positively mainly in-portal and peri-portal areas, showed the absence of detectable elastic fibres (Fig. 1B)

The patient improved progressively. Aminotransferase values fluctuated moderately, becoming normal in March 2001.

In September 2001 he was found to have markedly raised aminotransferases, up to 20 times the upper limit of normal. There was a relative lymphocytosis (Lymphocytes 52\%, Neutrophils 33\%). Anti-VCA/EBV $\mathrm{IgM}$ (EIA and Indirect Immunofluorescence) was positive. Other viral markers (HBV DNA, HCV RNA, HGV RNA) were negative on all occasions by PCR. Autoantibodies (ANCA, AMA, SMA, ENA, ANA, ANA-Hep2, LKM ) were all negative.

In February 2002 ANA (immunofluorescence) became positive, and ANA-Hep2 were detected at a title of 1:2560, with homogeneous pattern. ANCA,

Corresponding Author: D. Cabibi, Department of Human Pathology, Policlinico P. Giaccone I-90127 Palermo (Italy) Tel: 091-6553533 Fax: 0916553525 


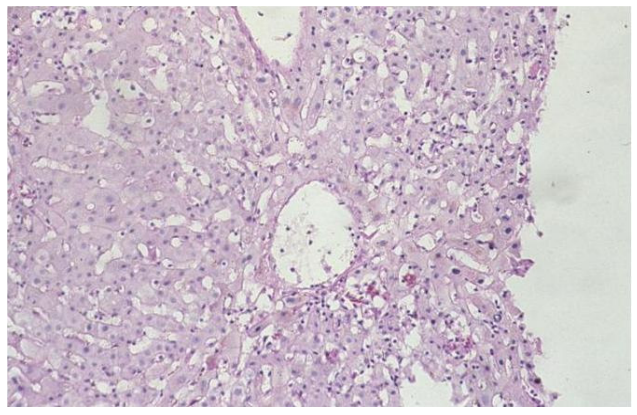

Fig. 1A: Acute hepatitis. Parenchymal damage in perivenular areas with spotty necrosis, livercell drop- out and infiltration by lymphocytes and macrophages (H-E stain 250x)

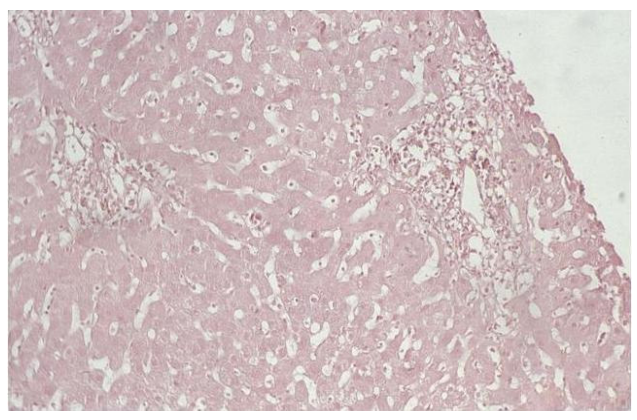

Fig. 1B: Acute hepatitis. No detectable elastic fibres in portal tracts by Shikata orcein staining

AMA, SMA, LKM, ENA (immunoblotting) and antiDNA (immunofluorescence) were negative. IgM anti$\mathrm{VCA} / \mathrm{EBV}$ (EIA) was negative, but IgG anti-VCA/EBV (EIA) and EBNA/EBV IgG were positive. By RT-PCR, EBV-DNA copy number in the peripheral mononuclear cells was negative. The high title positivity for ANA, negative on two previous occasions in May 2000 and March 2001, was considered significant for autoimmune disease (autoimmune hepatitis type 1) and a second liver biopsy was performed.

The histologic picture was now that of a chronic hepatitis, consistent with $\mathrm{AIH}$. There was a moderate interface hepatitis with periportal and lobular necroinflammatory activity. The portal tracts were expanded by a mononuclear infiltrate rich in plasma cells, the limiting plate was focally disrupted and the inflammatory process extended into the acinus. Scant small granulomas, a few macrophages PAS positiveDiastase resistant and some rosettes of hepatocytes were seen in the parenchyma (Fig. 2A). Unlike previous biopsy, now there was slight fibrosis containing elastic fibres detectable by Shikata orcein staining, limited to

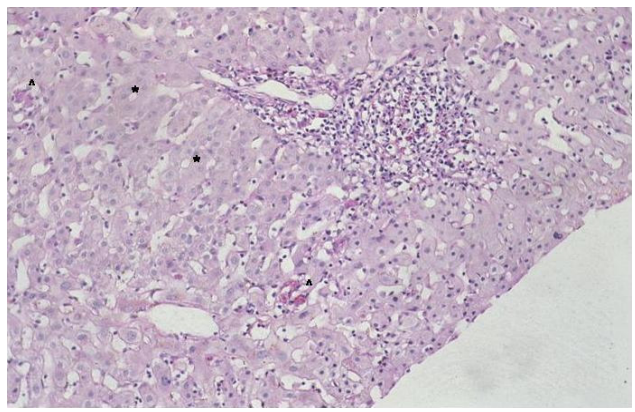

Fig. 2A: Chronic hepatitis consitent with AIH. Moderate periportal and lobular necroinflammatory activity, rosettes of hepatocytes, inflammatory infiltrate rich in plasma cells. Scant small granulomas in the parenchima (H-E stain 250x)

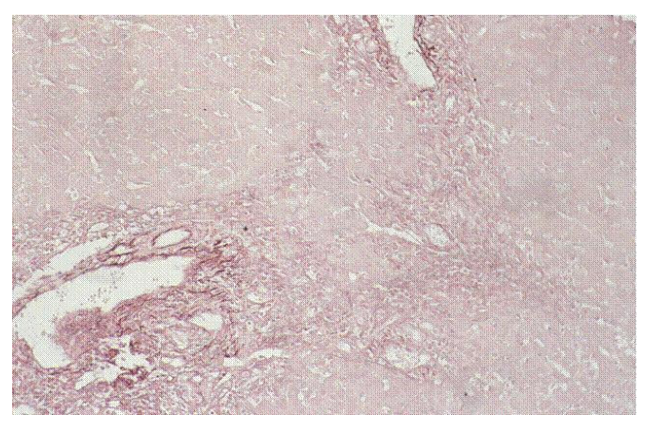

Fig. 2B: Slight portal and periportal fibrosis with detectable elastic fibres (Shikata orcein staining 250x)

portal tracts, while bridges of confluent necrosis were negative for elastic fibres (Fig. 2B). In situ hybridization assay was negative for EBV.

The patient was treated with methylprednisolone ( $37.5 \mathrm{mg}$ /day for 2 months reducing to $25 \mathrm{mg}$ /day) with rapid clinical and biochemical improvement. In October 2003 methylprednisolone was reduced to $5 \mathrm{mg} / \mathrm{day}$ and azathioprine $(1 \mathrm{mg} / \mathrm{Kg} /$ day $)$ added. Immunosuppressive therapy was gradually reduced, and finally stopped in October 2004. Serum aminotransferases returned into normal values and ANA-Hep2 were detected at a titre of 1:80 (with homogeneous pattern).

At the time of writing the patient is clinically well, and off all therapy. He is working at fully capacity.

\section{DISCUSSION}

Autoimmune hepatitis is usually an aggressive disease that leads to cirrhosis and has a mortality of up to 50 percent within 2 to 4 years. Exacerbations after 
cessation of treatment are frequent $(>50 \%)$ and indefinite therapy is often required. Signs indicating an unfavourable prognosis include high inflammatory activity and severe fibrosis on liver biopsy. Immunosuppressive therapy with corticosteroids (with or without azathioprine) results in remission of inflammation, but usually does not affect fibrogenic activity with its potential for cirrhosis ${ }^{[10]}$.

The first liver biopsy of our patient, in May 2000, showied the features of acute hepatitis. Due to the positivity for Anti-HEV, it was labeled as acute hepatitis type E.

All abnormal laboratory parameters improved to normal levels within 10 months but in September 2001, a new marked increase of aminotransferases, prompted us to perform a second biopsy, on February 2002. The new liver specimen showed findings that were in keeping with a chronic active hepatitis. They consisting of portal tracts expanded by a mononuclear infiltrate rich in plasma cells, a moderate interface hepatitis with periportal and lobular necroinflammatory activity, some rosettes of hepatocytes and mild portal and peri-portal fibrosis containing elastic fibres.

In 1999 the International Autoimmune Hepatitis Group $^{[11]}$ stated that there are no morphologic features patognomonic of autoimmune hepatitis, but the characteristic histologic picture is that of an interface hepatitis with a predominantly lymphoplasmacytic infiltrate, often with the formation of liver cell rosettes. They numerically evalued histological and clinical parameters to make a diagnosis of autoimmune hepatitis establishing a score $>15$ for cases before treament and $>17$ for cases after treatment to be necessary for a definite diagnosis of AIH. Our patient fulfilled most of the clinical and histological features of the revised scoring system and reached a score $>15$ pretreatment and $>17$ post-treatment, so a diagnosis of cronic hepatitis consistent with AIH, was made.

Autoimmune hepatitis may be induced by an external trigger in genetically predisposed individuals $^{[6]}$. Hepatitis $A$ virus, hepatitis $C$ virus, measles virus, cytomegalovirus and Epstein-Barr virus have all been suggested as potential triggers ${ }^{[1,2,3,4,6,7,8,9,10]}$. On the contrary, previous studies have indicated no relationship between HEV infection and autoimmune hepatitis ${ }^{[12,13]}$.

Vento et $a l^{[6]}$ suggested that in susceptible individuals, due to a defect in suppressor-inducer $\mathrm{T}$ lymphocytes specifically controlling immune responses to the asialoglycoprotein receptor (an antigen expressed on the hepatocyte surface), after EBV infection antibodies to this autoantigen increased and autoimmune hepatitis developed within 4 months.
In our patient the long time (more than 20 months) from acute hepatitis $\mathrm{E}$ as well as the short time betwen EBV infection and the appearance of autoantibodies (about 4 months, as reported by Vento et al.) make EBV to be the more probable trigger of the autoimmune disease. It is noteworthy that EBV-related autoimmune hepatitis need to be differentiated from primary EBV hepatitis with features of autoimmunity.

Recently, Chiba et al. ${ }^{[14]}$ reported a case of chronic active Epstein-Barr virus infection mimicking autoimmune hepatitis with fatal outcome after immunosoppressive treatment, and they underlined that hepatic involvement of chronic active EBV infection should be considered as differential diagnosis in cases showing liver dysfunction with clinical and biochemical features observed in AIH. On the contrary, Nakajima et al. ${ }^{[15]}$ reported a case of autoimmune hepatitis with favourable outcome that needed to be differentiated from EBV hepatitis in which they stressed the importance of the histology of liver biopsy specimen revealing interface hepatitis, dense mononuclear cell infiltrates, mild fibrosis, and negative for EBV in situ hybridization assay indicating AIH and not EBV-associated hepatitis.

We think that in our case, the time elapsing between EBV infection and clinical presentation of AIH with the decrease of IgM anti-EBV levels at the time of onset of autoantibodies, the absence of EBVDNA copy number in the peripheral mononuclear cells, the histological features of the liver biopsy specimen and the negativity of EBV in situ hybridization assay do not favor a direct pathogenetic role of persistent EBV infection, but suggest an immune-mediated mechanism in keeping with the hypothesis of Vento et al. ${ }^{[6]}$. AIH usually is as an aggressive disease leading to cirrhosis and with a mortality of up to 50 percent within 2 to 4 years and for which indefinite therapy is often required, the unfavorauble outcome of which is related to high inflammatory activity and severe fibrosis on liver biopsy. To our knowledge, this is the second case reported in the literature of EBV-related autoimmune hepatitis with complete clinical recovery and failure to relapse after cessation of therapy after 2 years followup without treatment.We can hypothesize that the favourable outcome in our patient may be related to the moderate degree of inflammation and to the low level of fibrosis at the early stage of the disease. Moreover, we would underline that the different outcome between EBV-related autoimmune hepatitis and primary EBV hepatitis with features of autoimmunity, the latter sometimes showing unfavourable prognosis after immunosoppressive treatment ${ }^{[14]}$, could be related to an increase of the direct pathogenetic effect of the virus 
after the immunosoppression. On the contrary, in EBVrelated autoimmune hepatitis the immunosoppressive treatment could be able to recover the disease with no adverse consequences.

Finally EBV-related AIH could have a more favourable prognosis than the most of $\mathrm{AIH}$ cases in which the trigger is unknown. Studies of a larger number of cases are desirable with a view to establishing whether there is a relationship between the nature of the trigger for $\mathrm{AIH}$ and its outcome.

\section{REFERENCES}

1. Vento, S., T. Garofalo, G. Di Perri, L. Dolci, E. Concia and D. Bassetti, 1991. Identification of hepatitis A virus as a trigger for autoimmune chronic type 1 in susceptible individuals. Lancet. 337: 1183-1187.

2. Huppertz, H.I., U. Treichel, A.M. Gassel, R. Jeschke and K.H. Meyer zum Buschenfelde, 1995. Autoimmune hepatitis following hepatitis A virus infection. J. Hepatol., 23: 204-208.

3. Rahaman, S.M., P. Chira and R.S. Koff, 1994. Idiopathic autoimmune chronic hepatitis triggered by hepatitis A. Am. J. Gastroenterol., 89: 106-108.

4. Leri, S. Paparo Barbaro, M.T. Sinopoli, V. Marcelli, F.G. Derosa and D. De Luca, 1996. Type 2 autoimmune hepatitis and hepatitis $\mathrm{C}$ viraemia. Riv. Eur. Sci. Med. Farmacol., 18: 3-6.

5. Vento, S., F. Cainelli, T. Ferraro and E. Concia, 1996. Autoimmune hepatitis type 1 after measles AJG: 12: 2618-2620.

6. Vento, S., L. Guella, F. Mirandola, F. Cainelli, G. Di Perri, M. Solbiati, T. Ferraro and E. Concia, 1995. Epstein-Barr virus as a trigger for autoimmune hepatitis in susceptible individuals. Lancet, 346: 608-9.

7. Manns, M.P., 1997. Hepatotropic viruses and autoimmunity. J. Viral. Hepat., 1997, 4:7-10.
8. Kojima, K., R. Nagayama, S. Hirama, T. Maeda, H. Takilawa, K. Miyake, M. Yamanaka and J. Shiga, 1999. Epstein-Barr virus infection resembling autoimmune hepatitis with lactate dehydrogenase and alkaline phosphatase anomaly. J. Gastroenterol., 34: 706-1.

9. Drebber, U., M. Odenthal, V. Dries, P. Schrimacher, H.P. Dienes, 2001. Diagnosis of seronegative chronic hepatitis in tissue. Pathologe, 22: 36-74.

10. Renner, E.L., 1993. Autoimmune chronic active hepatitis. Ther Umsch 50: 100-109.

11. International Autoimmune Hepatitis Group 1999 International autoimmune hepatitis group report: Review of criteria for diagnosis of autoimmune hepatitis. J. Hepatol., 31: 929-938.

12. Walter, E., 1994. Hepatitis E-epidemiology and clinical aspects. Schweiz Rundsch Med Prax, 83: 1008-1010.

13. Knodle, B., J. Hiller, C.C. Loliger and L. Kuhnl, 1994. Hepatitis E antibodies in blood donors, hemodialysis patients and in normal people. Beitr Infusionsther Transfusionsmed, 32: 124-127.

14. Chiba, T., S. Goto, O. Yokosuka, F. Imazeki, M. Tanaka, K. Fukai, Y. Takahashi, H. Tsujimura and H. Saisho 2004. Fatal chronic active EpsteinBarr virus infection mimicking autoimmune hepatitis. Eur. J. Gastroenterol. Hepatol., 16 (2): 225-228.

15. Nakajima, S., H. Umebayashi, R. Kurosawa, T. Imagawa, S. Katakura, M. Mori, Y. Aihara, S. Yokota, T. Sogo, A. Inui, T. Fujisawa and N. Hanzawa, 2005. A case of autoimmune hepatitis needed to be differentiated from EBV hepatitis. In: that the histology of liver biopsy specimen was useful for diagnosis Nihon Rinsho Meneki Gakkai Kaishi. 28 (3): 154-158. 